\title{
Welchen Effekt haben Bond-Ratingänderungen und deren Begründung auf den Shareholder Value?
}
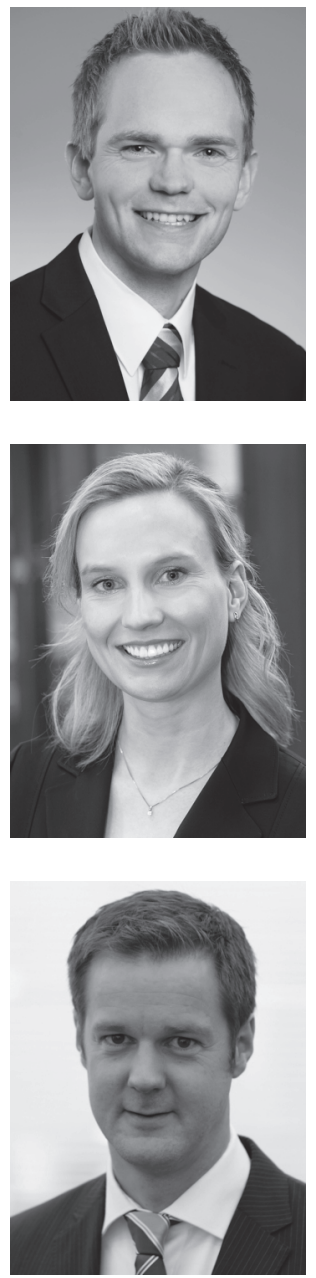

Steffen Biermann, Corinna Ewelt-Knauer und Andreas Wömpener

Rating, Ratingagenturen, Unternehmenswert, Ereignisstudie

Rating, Rating Agencies, Corporate Value, Event Study

Mit der vorliegenden Ereignisstudie können die theoretisch postulierten Wirkungen von Ratingmeldungen (Überprüfungen sowie Änderungen) erstmalig sowohl hinsichtlich der Performance als auch der Kapitalstruktur bestätigt werden. So kann gezeigt werden, dass eine performancebedingte Heraufstufung zu einem steigenden Shareholder Value führt, während eine Minderung des Verschuldungsgrades mit negativen abnormalen Renditen verbunden ist. Bei Herabstufungen können die genau reziproken Wirkungszusammenhänge festgestellt werden. Im Rahmen einer multivariaten Analyse werden zudem verschiedene Einflussfaktoren auf die abnormale Rendite analysiert. Für die Untersuchung werden sämtliche Ratingmeldungen der Agentur Moody's für Unternehmen des HDAX im Zeitraum von 2000 bis 2010 ausgewertet.

Our research verifies Option Price theory in the context of rating notices (review notices and notices of rating changes). We add to the extant research by differentiating between performance and capital structure related notices. We show that performance related upgrades imply shareholder value gains while a decreasing leverage is associated with negative abnormal returns. Downgrades lead to contrarian results. In addition, we examine different influencing factors in the context of a multivariate analysis.

\section{Einführung}

Spätestens seit der Staatsschuldenkrise werden Ratingagenturen in der Öffentlichkeit als äusserst einflussreich wahrgenommen. Fraglich ist jedoch, ob Ratingmeldungen in Form von angekündigten Ratingüberprüfungen oder von tatsächlichen Ratingänderungen auch den Shareholder Value des jeweiligen Unternehmens beeinflussen können. Grundsätzlich kann vermutet werden, dass eine Einschätzung über die Fähigkeit oder den Willen eines Unternehmens, seinen Zins- und Tilgungsleistungen vollständig und rechtzeitig nachzukommen, auch für Eigenkapitalgeber wertrelevant ist. Einen theoretischen Erklärungsansatz stellt dabei die optionspreistheoretische Kreditbewertung von 
Merton (1974) dar. Nach diesem Modell kann der Wert des Eigenkapitals als Wert einer europäischen Call-Option auf den Unternehmenswert interpretiert werden. Folglich profitieren ausschliesslich Anteilseigner von Unternehmenswertsteigerungen. Fremdkapitalgeber erhalten hingegen maximal den Kapitaldienst bestehend aus Nennwert der Verbindlichkeit nebst einhergehenden Zinszahlungen. Fällt der Wert des Unternehmens unter den Nennwert des Fremdkapitals, tragen allein die Fremdkapitalgeber die weiteren Verluste, wenn eine Haftungsbeschränkung der Anteilseigner unterstellt wird.

Der Wert des Fremdkapitals wird in der Praxis oftmals von Ratingagenturen beurteilt, wobei Wertschwankungen insbesondere aus zwei Sachverhalten rühren können. So können sich zum einen die Erfolgschancen des Unternehmens ändern, was die Zahlungsfähigkeit des Unternehmens beeinflussen könnte. Aus Perspektive der Eigenkapitalgeber dürfte sich in einem solchen Fall der Unternehmenswert ebenfalls ändern. Wird bspw. ein Unternehmen in seinem Rating aufgrund verschlechterter Performancechancen herabgestuft, sollte die Marktkapitalisierung, als Summe der diskontierten künftigen Zahlungsströme des Unternehmens, ebenfalls sinken. Zum anderen kann der Wert des Fremdkapitals durch eine Änderung der Kapitalstruktur des Unternehmens beeinflusst werden. Substituiert ein Unternehmen bspw. fortlaufend Eigenkapital durch Fremdkapital, so kann aufgrund eines ansteigenden Verschuldungsgrades der Wert des Fremdkapitals sinken, da die Gefahr besteht, dass das Unternehmen die Schulden nicht vollständig bedienen kann. Dies hätte die Konsequenz einer Abstufung durch die Ratingagenturen. Eine solche Veränderung der Kapitalstruktur würde indes zu einer Erhöhung des Marktwertes des Eigenkapitals führen. Diese Wirkung lässt sich im Folgenden theoretisch herleiten und empirisch nachweisen.

Der Beitrag gliedert sich wie folgt. In Kapitel 2 wird zunächst der theoretische Hintergrund skizziert, bevor daraus die Untersuchungshypothesen sowohl für den univariaten als auch den multivariaten Kontext abgeleitet werden. In Kapitel 3 wird das konkrete Design der Ereignisstudie nebst der entsprechenden Datengrundlage dargestellt. In Abschnitt 4 werden die Ergebnisse der Analyse vorgestellt und in Abschnitt 5 zusammenhängend erörtert. Ferner werden die Limitationen der Studie sowie der verbleibende Forschungsbedarf diskutiert.

\section{Herleitung der Hypothesen der Untersuchung}

\subsection{Theoretischer Hintergrund und Gang der Untersuchung}

Für die Zwecke dieser Studie sind Ratingmeldungen aufgrund einer veränderten Unternehmensperformance einerseits sowie einer veränderten Kapitalstruktur andererseits zu differenzieren. Die Wirkung von Ratingänderungen aufgrund veränderter Unternehmensperformance bedarf keiner umfangreichen theoretischen Herleitung: Eine Steigerung des Eigenkapitalmarktwertes aufgrund einer verbesserten Performance ist mit dem grundlegenden DCF-Ansatz zu begründen. Komplexer ist hingegen die Wirkung einer Ratingmeldung aufgrund einer Kapitalstrukturänderung.

Ein theoretischer Erklärungsansatz ergibt sich durch die unveränderte Nominalverzinsung des bestehenden Fremdkapitals in Verbindung mit der Risikosteigerung bei steigendem Verschuldungsgrad. Folglich kommt es zu einem zusätzlichen Werttransfer von den bestehenden Fremdkapitalgebern an die Residualgewinnempfänger, die Eigenkapitalgeber. Dieser Werttransfer lässt sich mit Hilfe der optionspreistheoretischen Kreditbewertung von Merton (1974) herleiten. Nach diesem Modell kann der Marktwert des Eigenkapitals 
als Wert einer europäischen Call-Option auf den Unternehmenswert interpretiert werden, wobei sich der Unternehmenswert aus dem Wert des Eigen- sowie dem Wert des Fremdkapitals zusammensetzt. Liegt der Unternehmenswert über dem Nominalwert des Fremdkapitals, wird der Eigentümer die Call-Option ausüben. Der Fremdkapitalgeber erhält in diesem Fall den Nominalwert seines gewährten Kapitals zurück. Liegt hingegen der Unternehmenswert unter dem Nominalwert des Fremdkapitals, lässt der Eigenkapitalgeber die Option verfallen, und die Eigenkapitaltitel sind wertlos. Der Wert des Fremdkapitals entspricht sodann dem Unternehmenswert und kann maximal den Nominalwert des Fremdkapitals betragen. Folglich profitieren ausschliesslich Anteilseigner von möglichen Unternehmenswertsteigerungen. Wird demnach Eigenkapital durch Fremdkapital substituiert, steigt für die Eigenkapitalgeber der Erwartungswert des Gewinns. Für die Fremdkapitalgeber steigt ausschliesslich das Risiko, da der Puffer bis zur Ausübung der Option (das Eigenkapital) sinkt. Daher sollte der Börsenkurs im Fall der kapitalstrukturbedingten Herabstufung mit positiven abnormalen Renditen reagieren. Zusammenfassend sollten kapitalstrukturbedingte Ratingmeldungen einen genau gegenläufigen Effekt auf den Börsenkurs haben, als jene Meldungen, die sich auf die Unternehmensperformance beziehen.

Insolvenzkosten, die realwirtschaftliche Aspekte umfassen, wie etwa Umsatzrückgang durch Stigmatisierung von Produkten oder Dienstleistungen, der Verlust kompetenter Mitarbeiter oder etwa Beratungs- und Restrukturierungskosten, sind im zitierten Modell von Merton (1974) nicht erfasst. Sie werden im Rahmen dieses Beitrags nicht als kapitalstrukturbedingt, sondern als performancebedingt interpretiert und entsprechend bei der Prüfung der Hypothesen berücksichtigt.

Die vorliegende Ereignisstudie untersucht diese theoretische Wirkungsweise für den deutschen Kapitalmarkt. Dafür werden sämtliche Ratingmeldungen der Agentur Moody's ausgewertet, die zwischen dem 1.1.2000 und dem 30.9.2010 zu Unternehmen des HDAX veröffentlicht wurden. ${ }^{1}$ Konkret umfasst die bereinigte Stichprobe 151 Meldungen von 38 Unternehmen. Für diese Meldungen wird in einem ersten Schritt analysiert, ob kurz vor oder kurz nach Veröffentlichung abnormale Renditen am Kapitalmarkt beobachtet werden können. In einem zweiten Schritt wird geprüft, ob diese abnormalen Renditen durch Ratingänderungen respektive angekündigte Ratingüberprüfungen insgesamt erklärt werden können. Neben einer pauschalen Analyse der Ratingmeldungen wird mit Blick auf die Optionspreistheorie zudem nach dem Grund der Ratingmeldung differenziert, wobei in performance- respektive kapitalstrukturbedingte Meldungen unterschieden wird. Die Signifikanz der Untersuchungsergebnisse wird mit parametrischen sowie nicht-parametrischen Tests überprüft. In einem dritten Schritt möchte die vorliegende Studie mithilfe eines multivariaten Modells die Einflussfaktoren auf die Höhe der abnormalen Rendite bei Ratingänderungen analysieren. Dabei werden als unabhängige Variablen das Ausgangsrating, der Umfang der Ratingänderung, das Über- bzw. Unterschreiten einer gewissen Investment Grade-Grenze und die Anzahl der beobachtenden Analysten genauso berücksichtigt wie die Tatsache, ob einer Änderung eine entsprechend angekündigte Überprüfung vorausgegangen ist. Die Signifikanz dieser Einflüsse wird auf Basis der t-Tests getestet, während die

1 Eine Beschränkung auf die Agentur Moody's erleichtert die Unterteilung in die Kategorien Performance und Kapitalstruktur. Ausserdem fördert dies die Vergleichbarkeit zu den Studien von Goh/Ederington (1993) und Imbierowicz/Wabrenburg (2009), die ebenfalls ausschliesslich Ratingmeldungen von Moody's verwenden. 
Güte des Modells über das adjustierte $\mathrm{R}^{2}$ gemessen wird. Mithilfe des F-Tests werden zudem die Unterschiede zwischen den untersuchten Populationen beurteilt.

\section{2 Überblick empirischer Studien zum Informationsgehalt von Ratingmeldungen}

Diese Studie führt verschiedene Aspekte zusammen, die zu einem Teil bereits in früheren Studien angelegt sind. So geht die grundlegende Fragestellung hinsichtlich des Einflusses von Ratingänderungen auf Aktienkurse auf Holthausen/Leftwich (1986) zurück, die USamerikanische Ratingmeldungen von 1977-1982 auswerten. Eine ähnliche Studie aller Ratingmeldungen zwischen 1970 und 1997 wurde von Dichev/Piotroski (2001) durchgeführt. Goh/Ederington (1993) differenzieren erstmalig hinsichtlich der Begründung für die Ratingänderungen, indem sie US-amerikanische Ratingänderungen der Jahre 1984-1986 der Agentur Moody's analysieren. In der Studie Gob/Ederington (1999) erweitern sie den Betrachtungszeitraum sowie das multivariate Modell zur Erklärung der abnormalen Rendite. Norden/Weber (2004) werten in einer weltweiten Studie den Informationswert sämtlicher Ratingänderungen von 2000-2002 der grossen Ratingagenturen Moody’s, S\&P sowie Fitch aus. Die Bedeutung des Ausgangsratings für den Informationsgehalt des Ratings stellen Jorion/Zhang (2007) heraus. Imbierowicz/Wabrenburg (2009) analysieren die Wirkung von Ratingmeldungen anhand weltweiter Meldungen der Ratingagentur Moody's für den Zeitraum 2001-2007. Bannier/Hirsch (2010) untersuchen, anhand von Daten der Ratingagentur Moody's aus dem Zeitraum zwischen 1982 und 2004, unter anderem den Einfluss einer vorherigen Ratingüberprüfung auf den Informationswert der Ratingänderung. Die folgende Tabelle 1 fasst die Ergebnisse dieser Studien vor dem Hintergrund der hier aufgeworfenen Fragestellung zusammen. Insbesondere wird erfasst, ob Ratingänderungen und angekündigte Ratingüberprüfungen gesondert analysiert werden, respektive ob hinsichtlich des Grundes (Kapitalstruktur versus Performance) unterschieden wird. Zudem wird dokumentiert, falls diese Studien neben einer univariaten Analyse zudem einen multivariaten Kontext aufgreifen.

\begin{tabular}{|c|c|c|c|c|c|}
\hline & \multicolumn{2}{|c|}{ Herabstufung } & \multicolumn{2}{|c|}{ Heraufstufung } & \multirow{2}{*}{$\begin{array}{l}\text { Multivariate } \\
\text { Analyse der Ein- } \\
\text { flussfaktoren } \\
\text { abnormaler } \\
\text { Rendite }\end{array}$} \\
\hline & Ratingüberprüfung & Ratingänderung & Ratingüberprüfung & Ratingänderung & \\
\hline $\begin{array}{l}\text { Holthausen/ } \\
\text { Leftwich } \\
(1986) \\
1977-1982 \\
\text { USA } \\
\text { Moody's } \\
\text { SむP }\end{array}$ & $\begin{array}{l}\text { Insgesamt: } \\
\text { Signifikant negativ } \\
\text { Keine Unterschei- } \\
\text { dung in Perfor- } \\
\text { mance und Kapi- } \\
\text { talstruktur }\end{array}$ & $\begin{array}{l}\text { Insgesamt: } \\
\text { Signifikant negativ } \\
\text { Keine Unterschei- } \\
\text { dung in Perfor- } \\
\text { mance und Kapital- } \\
\text { struktur }\end{array}$ & $\begin{array}{l}\text { Insgesamt: } \\
\text { Signifikant positiv } \\
\text { Keine Unterschei- } \\
\text { dung in Perfor- } \\
\text { mance und Kapital- } \\
\text { struktur }\end{array}$ & $\begin{array}{l}\text { Insgesamt: } \\
\text { nicht-signifikant } \\
\text { Keine Unter- } \\
\text { scheidung in Per- } \\
\text { formance und } \\
\text { Kapitalstruktur }\end{array}$ & ja \\
\hline $\begin{array}{l}\text { Goh/ } \\
\text { Ederington } \\
(1993) \\
\text { 1984-1986 } \\
\text { USA } \\
\text { Moody's }\end{array}$ & $\begin{array}{l}\text { Keine Untersu- } \\
\text { chung von Rating- } \\
\text { überprüfungen }\end{array}$ & $\begin{array}{l}\text { Insgesamt: signifi- } \\
\text { kant negativ } \\
\text { Performance: signi- } \\
\text { fikant negativ } \\
\text { Kapitalstruktur: } \\
\text { nicht-signifikant } \\
\text { positiv }\end{array}$ & $\begin{array}{l}\text { Keine Untersuchung } \\
\text { von Ratingüberprü- } \\
\text { fungen }\end{array}$ & $\begin{array}{l}\text { Insgesamt: nicht- } \\
\text { signifikant } \\
\text { Keine Unter- } \\
\text { scheidung in Per- } \\
\text { formance und } \\
\text { Kapitalstruktur }\end{array}$ & ja \\
\hline
\end{tabular}




\begin{tabular}{|c|c|c|c|c|c|}
\hline & \multicolumn{2}{|c|}{ Herabstufung } & \multicolumn{2}{|c|}{ Heraufstufung } & \multirow{2}{*}{$\begin{array}{l}\text { Multivariate } \\
\text { Analyse der Ein- } \\
\text { flussfaktoren } \\
\text { abnormaler } \\
\text { Rendite }\end{array}$} \\
\hline & Ratingüberprüfung & Ratingänderung & Ratingüberprüfung & Ratingänderung & \\
\hline $\begin{array}{l}\text { Goh/ } \\
\text { Ederington } \\
(1999) \\
\text { 1984-1990 } \\
\text { USA } \\
\text { Moody's }\end{array}$ & $\begin{array}{l}\text { Keine Untersu- } \\
\text { chung von Rating- } \\
\text { überprüfungen }\end{array}$ & $\begin{array}{l}\text { Insgesamt: signifi- } \\
\text { kant negativ } \\
\text { Performance: signi- } \\
\text { fikant negativ }\end{array}$ & $\begin{array}{l}\text { Keine Untersuchung } \\
\text { von Ratingüberprü- } \\
\text { fungen }\end{array}$ & $\begin{array}{l}\text { Insgesamt: nicht } \\
\text { signifikant posi- } \\
\text { tiv } \\
\text { Performance: } \\
\text { nicht-signifikant } \\
\text { negativ }\end{array}$ & ja \\
\hline $\begin{array}{l}\text { Dichev/ } \\
\text { Piotroski } \\
\text { (2001) } \\
\text { 1970-1997 } \\
\text { USA } \\
\text { Moody's }\end{array}$ & $\begin{array}{l}\text { Keine Untersu- } \\
\text { chung von Rating- } \\
\text { überprüfungen }\end{array}$ & $\begin{array}{l}\text { Insgesamt: } \\
\text { Signifikant negativ } \\
\text { Keine Unterschei- } \\
\text { dung in Perfor- } \\
\text { mance und Kapital- } \\
\text { struktur }\end{array}$ & $\begin{array}{l}\text { Keine Untersuchung } \\
\text { von Ratingüberprü- } \\
\text { fungen }\end{array}$ & $\begin{array}{l}\text { Insgesamt: } \\
\text { Signifikant posi- } \\
\text { tiv } \\
\text { Keine Unter- } \\
\text { scheidung in Per- } \\
\text { formance und } \\
\text { Kapitalstruktur }\end{array}$ & nein \\
\hline $\begin{array}{l}\text { Gropp/ } \\
\text { Richards } \\
\text { (2001) } \\
\text { 1989-2000 } \\
\text { Banken: Eu- } \\
\text { ropa } \\
\text { Moody's } \\
\text { Sహ,P } \\
\text { Fitch }\end{array}$ & $\begin{array}{l}\text { Keine Untersu- } \\
\text { chung von Rating- } \\
\text { überprüfungen }\end{array}$ & $\begin{array}{l}\text { Insgesamt: signifi- } \\
\text { kant negativ } \\
\text { Performance: signi- } \\
\text { fikant negativ } \\
\text { Kapitalstruktur: si- } \\
\text { gnifikant positiv }\end{array}$ & $\begin{array}{l}\text { Keine Untersuchung } \\
\text { von Ratingüberprü- } \\
\text { fungen }\end{array}$ & $\begin{array}{l}\text { Insgesamt: nicht } \\
\text { signifikant } \\
\text { Performance: } \\
\text { nicht-signifikant } \\
\text { positiv } \\
\text { Kapitalstruktur: } \\
\text { signifikant posi- } \\
\text { tiv }\end{array}$ & nein \\
\hline $\begin{array}{l}\text { Norden/ } \\
\text { Weber (2004) } \\
\text { 2000-2002 } \\
\text { Welt } \\
\text { Moody's } \\
\text { SむP, Fitch }\end{array}$ & $\begin{array}{l}\text { Insgesamt: signifi- } \\
\text { kant negativ } \\
\text { Keine Unterschei- } \\
\text { dung in Perfor- } \\
\text { mance und Kapi- } \\
\text { talstruktur }\end{array}$ & $\begin{array}{l}\text { Insgesamt: signifi- } \\
\text { kant negativ } \\
\text { Keine Unterschei- } \\
\text { dung in Perfor- } \\
\text { mance und Kapital- } \\
\text { struktur }\end{array}$ & $\begin{array}{l}\text { Keine Untersuchung } \\
\text { von Upgrades }\end{array}$ & $\begin{array}{l}\text { Keine Untersu- } \\
\text { chung von Up- } \\
\text { grades }\end{array}$ & ja \\
\hline $\begin{array}{l}\text { Jorion/Zhang } \\
\text { (2007) } \\
1996-2002 \\
\text { USA } \\
\text { Moody's, } \\
\text { Sむ'P, Fitch }\end{array}$ & $\begin{array}{l}\text { Keine Untersu- } \\
\text { chung von Rating- } \\
\text { überprüfungen }\end{array}$ & $\begin{array}{l}\text { Insgesamt: } \\
\text { Signifikant negativ } \\
\text { Keine Unterschei- } \\
\text { dung in Perfor- } \\
\text { mance und Kapital- } \\
\text { struktur }\end{array}$ & $\begin{array}{l}\text { Keine Untersuchung } \\
\text { von Ratingüberprü- } \\
\text { fungen }\end{array}$ & $\begin{array}{l}\text { Insgesamt: } \\
\text { Signifikant posi- } \\
\text { tiv } \\
\text { Keine Unter- } \\
\text { scheidung in Per- } \\
\text { formance und } \\
\text { Kapitalstruktur }\end{array}$ & ja \\
\hline $\begin{array}{l}\text { Imbierowicz/ } \\
\text { Wabrenburg } \\
(2009) \\
2001-2007 \\
\text { Welt } \\
\text { Moody's }\end{array}$ & $\begin{array}{l}\text { Insgesamt: signifi- } \\
\text { kant negativ } \\
\text { Performance: signi- } \\
\text { fikant negativ } \\
\text { Kapitalstruktur: si- } \\
\text { gnifikant positiv }\end{array}$ & $\begin{array}{l}\text { Insgesamt: signifi- } \\
\text { kant negativ } \\
\text { Performance: signi- } \\
\text { fikant negativ } \\
\text { Kapitalstruktur: } \\
\text { nicht signifikant }\end{array}$ & $\begin{array}{l}\text { Insgesamt: signifi- } \\
\text { kant positiv } \\
\text { Keine Unterschei- } \\
\text { dung in Perfor- } \\
\text { mance und Kapital- } \\
\text { struktur }\end{array}$ & $\begin{array}{l}\text { Insgesamt: nicht- } \\
\text { signifikant posi- } \\
\text { tiv } \\
\text { Keine Unter- } \\
\text { scheidung in Per- } \\
\text { formance und } \\
\text { Kapitalstruktur }\end{array}$ & nein \\
\hline $\begin{array}{l}\text { Bannier/ } \\
\text { Hirsch } \\
(2010) \\
\text { 1982-2004 } \\
\text { USA } \\
\text { Moody's }\end{array}$ & $\begin{array}{l}\text { Herabstufungen } \\
\text { ohne vorherige } \\
\text { Überprüfung signi- } \\
\text { fikant und stärker } \\
\text { als mit Überprü- } \\
\text { fung. }\end{array}$ & $\begin{array}{l}\text { Insgesamt: signifi- } \\
\text { kant negativ } \\
\text { Keine Unterschei- } \\
\text { dung in Perfor- } \\
\text { mance und Kapital- } \\
\text { struktur }\end{array}$ & $\begin{array}{l}\text { Heraufstufungen } \\
\text { ohne vorherige } \\
\text { Überprüfung nicht } \\
\text { signifikant aber } \\
\text { stärker als mit } \\
\text { Überprüfung. }\end{array}$ & $\begin{array}{l}\text { Insgesamt: nicht- } \\
\text { signifikant } \\
\text { Keine Unter- } \\
\text { scheidung in Per- } \\
\text { formance und } \\
\text { Kapitalstruktur }\end{array}$ & ja \\
\hline
\end{tabular}

Tabelle 1: Studien zu Aktienmarktreaktionen aufgrund von Ratingmeldungen

Der aktuelle Stand der Forschung zeigt, dass Studien bislang vorwiegend Teilaspekte der Optionspreistheorie beleuchtet haben. So liegt noch keine Analyse vor, die sowohl für Herauf- als auch für Herabstufungen konsequent die beiden folgenden Untersuchungsdi- 
mensionen unterscheidet: (1) angekündigte Ratingüberprüfung versus Ratingänderung, (2) kapitalstrukturbedingte versus performancebedingte Ratingmeldung. Zudem kommen die vorliegenden Studien zu widersprüchlichen Ergebnissen hinsichtlich der praktischen Relevanz der Optionspreistheorie, was in einer Fokussierung auf die jeweiligen Teilbereiche begründet sein mag. So werden theoretisch für kapitalstrukturbedingte Herabstufungen positive abnormale Renditen geschlussfolgert. Ein solcher signifikant positiver Zusammenhang kann jedoch nur von Gropp/Richards (2001) gezeigt werden. Goh/Ederington (1993) können zwar einen positiven Wirkungszusammenhang belegen, wenngleich dieser als nicht signifikant deklariert werden muss. Imbierowicz/Wabrenburg (2009) finden unter anderem hingegen eine genau umgekehrte Wirkungsrichtung, indem sie signifikant negative Effekte feststellen müssen. Zudem existieren nur wenige Studien, die neben einer univariaten Analyse der abnormalen Rendite versuchen, Einflüsse auf die Höhe der abnormalen Rendite im multivariaten Kontext festzustellen. Abschliessend sei angemerkt, dass noch keine Studie vorliegt, die den deutschen Kapitalmarkt gesondert betrachtet. Vielmehr sind bislang Ratingmeldungen deutscher Unternehmen nur bei internationalen Studien undifferenziert berücksichtigt worden. Es müssen jedoch starke Bedenken angemeldet werden, ob die Ergebnisse der internationalen respektive der US-amerikanischen Untersuchungen uneingeschränkt auf den deutschen Markt übertragen werden können. So ist die Bedeutung von Ratings in Deutschland - wohl im Hinblick auf die weiterhin vorherrschende Dominanz von Bankkrediten anstelle von am Kapitalmarkt platzierten Anleihen als Fremdfinanzierungsinstrument - allgemein geringer, was die Einflüsse von Ratingmeldungen auf Eigenkapitalgeber einschränken könnte (Wieben 2004, 13; Wappenschmidt 2009, 17). Weiterhin stellen die grössere Bedeutung des Kapitalmarktes für Privatanleger und ein schärferes Haftungsumfeld in den USA bedeutende Unterschiede dar, die zu einer unterschiedlichen Kapitalmarktkommunikation der Unternehmen einerseits und andererseits zu einer unterschiedlichen Rezeption von Informationen seitens des Kapitalmarktes führen. Schlussendlich zeigt ein Vergleich der kumulierten Ausfallraten von Europa und Nordamerika hinsichtlich der einzelnen Ratingklassen deutliche Unterschiede. Während bspw. die kumulierte zehnjährige Ausfallrate bei der Kategorie Baa für Europa 11,41\% beträgt, beläuft sich diese für Nordamerika auf 22,59\%. Diese unterschiedlichen Ausfallwahrscheinlichkeiten trotz Einstufung in dieselbe Kategorie könnten zu unterschiedlichen Reaktionen bei den Eigenkapitalgebern führen.

\subsection{Hypothesen der Untersuchung}

Ratingmeldungen können zum einen auf einer veränderten (erwarteten) Unternehmensperformance gründen und zum anderen auf eine geänderte Kapitalstruktur zurückzuführen sein (Goh/Ederington 1993). Wird der Unternehmenswert als diskontierte Nettozahlungsströme, die das Unternehmen in Zukunft erwirtschaften wird, interpretiert, wird eine verschlechterte Performance zu geminderten Zahlungsströmen führen, was c. p. den Unternehmenswert negativ beeinflusst, et vice versa. Von einem solchen Rückgang des Unternehmenswertes wären Eigenkapitalgeber genauso betroffen wie Fremdkapitalgeber. Während für Fremdkapitalgeber die Gefahr steigt, dass das Unternehmen seinen Kapitaldienst nicht mehr bedienen kann, sinkt für Eigenkapitalgeber der erwartete Cash Flow. Folglich müssten Ratingänderungen hinsichtlich einer verschlechterten (verbesserten) Performance zu negativen (positiven) abnormalen Renditen führen. 
Ratingmeldungen aufgrund eines steigenden (sinkenden) Verschuldungsgrades dürften den Wert des Fremdkapitals negativ (positiv) beeinflussen, während sich c. p. der Marktwert des Eigenkapitals erhöht (mindert), da der Unternehmenswert aufgrund der unveränderten Zukunftserwartungen konstant bleibt. Folglich sollten Ratingherabstufungen aufgrund von Änderungen der Kapitalstruktur einen positiven Einfluss auf die Höhe der abnormalen Renditen haben, während kapitalstrukturbedingte Heraufstufungen die abnormale Rendite negativ beeinflussen sollten.

Insgesamt zeigt sich, dass Ratingmeldungen je nach Anlass entgegengesetzt auf die abnormalen Rendite wirken. Während bspw. eine Herabsetzung wegen verschlechterter Ertragsaussichten einen negativen Einfluss auf die abnormale Rendite ausübt, dürfte eine Herabsetzung des Ratings aufgrund einer geänderten Kapitalstruktur die abnormale Rendite erhöhen. Folglich können sich Effekte resultierend aus Ratingmeldungen überlagern.

Mit Blick auf die theoretisch abgeleiteten Wirkungen können demnach die folgenden Hypothesen aufgestellt werden:

\section{H1a: Performancebedingte Ratingänderungen bzw. -überprüfungen haben bei einer Herabstufung (Heraufstufung) einen negativen (positiven) Einfluss auf die Höhe der abnormalen Rendite.}

\section{H1b: Kapitalstrukturbedingte Ratingänderungen bzw. -überprüfungen haben bei einer Herabstufung (Heraufstufung) einen positiven (negativen) Einfluss auf die Höhe der abnormalen Rendite.}

Auf Basis einer multivariaten Analyse werden weitere Einflussfaktoren identifiziert, die auf die Höhe der abnormalen Rendite bei Herab- respektive Heraufstufungen wirken. So soll in einem ersten Schritt überprüft werden, ob die abnormalen Renditen unterschiedlich hoch sind, wenn bei einer Ratingänderung der Kapitalmarkt zuvor über eine entsprechende Ratingüberprüfung informiert wurde. So könnte vermutet werden, dass der Kapitalmarkt eine entsprechende Wirkung bereits bei Ankündigung (zumindest partiell) antizipiert (Bannier/Hirsch 2010). Folglich wäre der Effekt bei einer sodann folgenden tatsächlichen Ratingänderung geringer, als wenn dieser Änderung keine entsprechende Ankündigung vorausgegangen wäre. Auf diese Weise kann die Bereitschaft des Kapitalmarkts beurteilt werden, nicht-gesicherte Informationen bei der Preissetzung zu antizipieren, da eine Ratingüberprüfung nicht zwingend in einer tatsächlichen Herauf- respektive Herabsetzung münden muss. Konkret soll folgender Wirkungszusammenhang vermutet werden:

\section{H2: $\quad$ Ist der Ratingänderung eine angekündigte Ratingüberprüfung vorangegangen, ist der Einfluss auf die abnormale Rendite geringer, als wenn eine solche Überprüfung nicht an den Kapitalmarkt kommuniziert worden wäre.}

Mit Blick auf eine mögliche Risikoaversion der Eigenkapitalgeber könnte zudem gelten, dass die Höhe der abnormalen Rendite vom Ausgangsrating des Unternehmens beeinflusst wird. So ist zu vermuten, dass der Einfluss umso grösser ist, je schlechter das Ausgangsrating des Unternehmens ist (Jorion/Zhang 2007, 46-49). Konkret steigt die Insolvenzwahrscheinlichkeit - und damit der Verlust der Einlage - bei einer Verschlechterung des Ratings von Stufe zu Stufe. Folglich könnte geschlussfolgert werden, dass bei Herabstufungen 
von Unternehmen umso mehr Anteile veräussert werden, je schlechter das Unternehmen im Vorfeld geratet war. Da durch diese Veräusserungen der Kurswert der Aktie im Ereignisfenster sinken dürfte, müsste folglich gelten, dass je schlechter das Ausgangsrating ist, desto stärker wird die abnormale Rendite negativ beeinflusst. Umgekehrt sinkt die Ausfallwahrscheinlichkeit und damit die Wahrscheinlichkeit einer Unternehmensinsolvenz bei einer Heraufstufung umso stärker, je schlechter das Ausgangsrating ist. Folglich sollte bei einer Heraufstufung ein deutlich positiver Einfluss auf die abnormale Rendite festzustellen sein.

H3: Bei Herabstufungen wird die abnormale Rendite umso stärker negativ beeinflusst, je schlechter das Ausgangsrating ist. Bei Heraufstufungen wird die abnormale Rendite umso stärker positiv beeinflusst, je schlechter das Ausgangsrating ist.

\section{Design der empirischen Untersuchung}

\subsection{Untersuchungsmethodik}

Die Wirkung von Ratingmeldungen auf den Kapitalmarkt soll anhand des Ereignisstudienansatzes auf Basis abnormaler Renditen analysiert werden (Ball/Brown 1968; Fama et al. 1969). Dabei wird die am Ereignistag oder im Ereignisfenster tatsächlich erzielte Aktienrendite des betroffenen Unternehmens mit jener Rendite verglichen, die ohne dieses Ereignis zu erwarten gewesen wäre (Brown/Warner 1980, 207-208; Campbell et al. 1997, 150-152; Gerpott 2009, 214-216). Die Differenz wird als abnormale Rendite bezeichnet. Konkret gilt:

$$
A R_{i t}=R_{i t}-E\left(R_{i t}\right)
$$

mit: $\quad A R_{i t} \quad$ abnormale Rendite der Aktie i zum Zeitpunkt $\mathrm{t}$

$R_{i t} \quad$ beobachtete Rendite der Aktie i zum Zeitpunkt $\mathrm{t}$

$E\left(R_{i t}\right) \quad$ erwartete normale Rendite der Aktie i zum Zeitpunkt $\mathrm{t}$

Die beobachtete Rendite $R_{i t}$ kann entweder diskret oder stetig ermittelt werden, wobei im Rahmen von Ereignisstudien die diskrete Variante bevorzugt wird (Röder 1999, 13; Gerpott 2009, 216):

$R_{i t}=\frac{A_{i t}-A_{i t-1}}{A_{i t-1}} \times 100$

mit: $A_{i t} \quad$ Börsenschlusskurs der Aktie i am Tag t

Unter der Prämisse der Stationarität kann die erwartete normale Rendite $E\left(R_{i t}\right)$ anhand des sog. Marktmodels geschätzt werden (Gerpott 2009, 216). Bei diesem Einfaktormodell wird die Marktrendite als erklärende Variable im Rahmen einer Kleinste-Quadrate-Regression verwendet (Campbell et al. 1997, 158 f; MacKinlay 1997, 20; McWilliams/Siegel 1997, 628-630; Gerpott 2009, 215-217), was mithilfe der folgenden Gleichung verdeutlicht werden kann: 


$$
E\left(R_{i t}\right)=\alpha_{i}+\beta_{i} R_{M t}+\varepsilon_{i t}
$$

mit: $\quad \alpha_{i} \quad$ unsystematische Renditekonstante der Aktie i

$\beta_{i} \quad$ Abhängigkeitsfaktor der Aktienrendite i von der systematischen Marktrendite

$R_{M t} \quad$ Marktrendite zum Zeitpunkt t

$\varepsilon_{i t} \quad$ Störterm der Aktie i zum Zeitpunkt t

Die Regressionsparameter $\alpha_{\mathrm{i}}$ und $\beta_{\mathrm{i}}$ werden anhand der linearen Regression für jede Beobachtung bestimmt. Während $\alpha_{\mathrm{i}}$ die unsystematische Renditekonstante einer Aktie darstellt, beschreibt $\beta_{\mathrm{i}}$ die Intensität der Abhängigkeit der Aktienrendite von der Marktrendite. Theoretisch stellt diese Marktrendite die Rendite des Marktportfolios dar, das sämtliche Kapitalanlagen beinhaltet. Praktisch empfehlen Brown/Warner (1980) einen Index derjenigen Unternehmen zu verwenden, die in die Untersuchung einbezogen sind (Brown/Warner 1980, 243). Da hier vornehmlich Unternehmen des HDAX ausgewertet werden, wird vereinfachend dieser Index verwendet. Konkret erfolgt die Regression anhand von Renditedaten der 250 vorherigen Handelstage, um mögliche saisonale Schwankungen auszugleichen (Gerpott 2009, 217). Die Voraussetzungen für eine solche lineare Regression werden vom zugrundeliegenden Datensatz erfüllt, so dass eine unverzerrte und effiziente Schätzung der Modellparameter möglich ist (Backhaus et al. 2011, 84-86). So weist die Störgrösse einen Erwartungswert von Null auf, die Korrelation zwischen der erklärenden Variablen und der Störgrösse beträgt ebenfalls Null. Hinweise auf Heteroskedaszität konnten nicht gefunden werden und die Störgrösse ist normalverteilt.

Darauf aufbauend muss die abnormale Rendite als Differenz aus tatsächlicher Rendite und erwarteter normaler Rendite innerhalb des Ereignisfensters ermittelt werden. Dabei soll das Ereignisfenster so kurz wie möglich gewählt werden, damit diese Rendite nicht von weiteren kursbeeinflussenden Effekten verzerrt wird. Dennoch sollte es so lang wie nötig sein, um den Effekt vollständig abzubilden (McWilliams/Siegel 1997, 636). Ratingmeldungen können taggenau terminiert werden, da der Tag der Bekanntgabe der Meldung sowie die Meldung selbst auf der Homepage der Agentur abrufbar sind. ${ }^{2}$ Folglich ist ein kurzes Ereignisfenster von wenigen Tagen ausreichend. Konkret werden hier mit $[0,+1]$, $[-1,+1]$ und $[-2,+2]$ drei unterschiedlich lange Ereignisfenster untersucht. Durch einen Beginn von bis zu zwei Tagen vor dem Ereignistag können eventuelle vorgelagerte Effekte gemessen werden. Um eine eventuell verzögerte Informationsverarbeitung zu erfassen, schliesst ein Ereignisfenster erst zwei Tage nach der Ratingmeldung. Mathematisch ist die Regression zur Bestimmung der normalen Rendite aus Formel (3) in Formel (1) einzusetzen. Konkret wird die errechnete normale Rendite sowie die beobachtete Rendite der jeweiligen Aktie zum entsprechenden Betrachtungszeitpunkt in die folgende Formel eingesetzt, so dass gilt:

$A R_{i t}=R_{i t}-\alpha_{i}-\beta_{i} R_{M t}$

Um die Kapitalmarktreaktion des Ereignisses am Ereignistag bestimmen zu können, ist die durchschnittliche abnormale Rendite der Stichprobe für diesen Tag zu bestimmen:

2 Die Ratingmeldungen sind auf der Homepage www.Moodys.com frei zugänglich. 
$\overline{A R}_{t}=\frac{1}{N} \sum_{i=1}^{N} A R_{i t}$

mit: $\quad \overline{A R}_{t} \quad$ durchschnittliche abnormale Rendite zum Zeitpunkt $\mathrm{t}$

$N \quad$ Anzahl der Beobachtungen in der Stichprobe

Bei der Untersuchung eines Ereignisfensters wird die kumulierte durchschnittliche abnormale Rendite über diesen Zeitraum benötigt:

$\overline{\operatorname{KAR}_{v, \omega}}=\sum_{t=\nu}^{\omega} \overline{A R_{t}}$

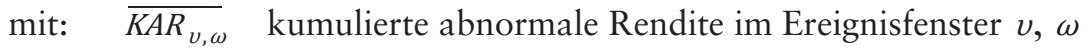

$v \quad$ erster Tag des Ereignisfensters

$\omega \quad$ letzter Tag des Ereignisfensters

Die Einflüsse auf die abnormale Rendite werden in einem ersten Schritt mit einem univariaten Modell geprüft. Mittels eines multivariaten Modells werden in einem zweiten Schritt die Einflüsse weiterer Variablen auf die abnormale Rendite analysiert. Die Signifikanz dieser Einflüsse wird mittels parametrischer sowie nicht-parametrischer Verfahren getestet. Während als parametrisches Verfahren der t-Test nach Brown/Warner angewendet wird, wird als nicht-parametrischer Test zum einen das Rangplatzverfahren nach Corrado eingesetzt, da dieses besonders für kleine Stichproben geeignet ist (Corrado 1989, 391 f.; Entrup 1995, 164). Jedoch weist das Corrado Verfahren beim Ablehnen nicht signifikanter Ereignisse Schwächen auf (Cowan 1992, 351 f.). Daher wird zusätzlich ein Vorzeichentest durchgeführt.

\subsection{Datengrundlage}

Insgesamt wurden sämtliche Meldungen der Agentur Moody's erfasst, die zwischen dem 1.1.2000 und dem 30.9.2010 zu Unternehmen des HDAX veröffentlicht wurden. Konkret enthält die unbereinigte Stichprobe 228 Meldungen. Bereinigungen wurden zum einen vorgenommen für überlappende Ereignisse, die in den drei Tagen vor sowie den drei Tagen nach der Bekanntgabe der Ratingmeldungen liegen und erheblichen Einfluss auf die abnormale Rendite ausüben. Um diese Ereignisse zu identifizieren, wurden Meldungen der relevantesten grossen (Wirtschafts-) Zeitungen durchgesehen sowie die Homepage der jeweiligen Unternehmen untersucht. Zum anderen waren Bereinigungen aufgrund fehlender Daten notwendig. So muss das Unternehmen 260 Tage vor der Ratingmeldung börsennotiert sein, um eine normale Rendite der Aktie ermitteln zu können. Die adjustierten Aktienkurse nebst Marktkapitalisierung der betroffenen Unternehmen sowie die Notierungen des Referenzindex HDAX werden der Datenbank Thomsen Reuters Datastream entnommen. Die Anzahl der Analysten, die das jeweilige Unternehmen beobachten, werden anhand der Datenbank FactSet bestimmt. Schlussendlich umfasst die bereinigte Stichprobe 151 Meldungen von 38 Unternehmen. Pro Unternehmen ergeben sich demnach durchschnittlich 3,97 nicht durch andere Effekte verzerrte Meldungen in einem Zeitraum von knapp zehn Jahren. Die Anzahl der Meldungen pro Unternehmen variiert zwischen einer Meldung und 14 Meldungen. Dabei ist zu beachten, dass die Anzahl der Meldungen nicht 
unbedingt auf die Grösse der Unternehmen zurückzuführen ist. So weisen die Unternehmen des MDAX sogar leicht mehr Meldungen pro Unternehmen auf als jene, die im DAX notiert sind (vgl. Tabelle 2).

\begin{tabular}{|c|c|c|c|c|}
\hline & DAX & MDAX & TecDAX & MK 100 \\
\hline Anzahl Unternehmen & 22 & 13 & 1 & 2 \\
\hline Anzahl Meldungen & 88 & 55 & 1 & 7 \\
\hline Anteil Unternehmen & $57,9 \%$ & $34,2 \%$ & $2,6 \%$ & $5,3 \%$ \\
\hline Anteil Meldungen & $58,3 \%$ & $36,4 \%$ & $0,7 \%$ & $4,6 \%$ \\
\hline Anzahl Meldungen pro Unternehmen & 4 & 4,2 & 1 & 3,5 \\
\hline \multicolumn{5}{|c|}{$\begin{array}{l}\text { Die Tabelle zeigt die Anzahl der Unternehmen, die in den einbezogenen Indizes von Ratingmeldungen be- } \\
\text { troffen sind und nicht aufgrund von Datenmangel ausgeschlossen wurden, sowie die Anzahl an Ratingmel- } \\
\text { dungen pro Index im Betrachtungszeitraum. Zudem wird der relative Anteil an Unternehmen und an Mel- } \\
\text { dungen pro Index angegeben. Die letzte Zeile zeigt die durchschnittliche Anzahl der Meldungen pro Unter- } \\
\text { nehmen. Mit MK } 100 \text { sind die Unternehmen bezeichnet, die nicht im HDAX notiert sind, aber nach } \\
\text { Marktkapitalisierung zu den einhundert grössten deutschen Unternehmen gehören. }\end{array}$} \\
\hline
\end{tabular}

Tabelle 2: Verteilung der Ratingmeldungen auf die einbezogenen Indizes

Tabelle 3 zeigt die Verteilung der Ratingmeldungen über den Betrachtungszeitraum sowie mit Blick auf die unterschiedlichen Ratingklassen.

\begin{tabular}{l|ccccccccccccc}
\hline \multicolumn{1}{c}{} & 2000 & 2001 & 2002 & 2003 & 2004 & 2005 & 2006 & 2007 & 2008 & 2009 & 2010 & Summe & $\%$ \\
\hline AAA - Aa & 2 & 7 & 2 & 4 & 0 & 1 & 0 & 0 & 0 & 2 & 1 & 19 & 12,6 \\
A & 2 & 7 & 4 & 7 & 6 & 1 & 3 & 2 & 3 & 6 & 2 & 43 & 28,5 \\
Baa & 1 & 3 & 6 & 11 & 7 & 3 & 1 & 6 & 8 & 5 & 2 & 53 & 35,1 \\
Ba & 0 & 1 & 0 & 1 & 4 & 3 & 5 & 6 & 2 & 5 & 0 & 27 & 17,9 \\
B - C & 0 & 0 & 0 & 0 & 0 & 2 & 1 & 1 & 0 & 5 & 0 & 9 & 6,0 \\
\hline Summe & 5 & 18 & 12 & 23 & 17 & 10 & 10 & 15 & 13 & 23 & 5 & 151 & \\
$\%$ & 3,3 & 11,9 & 7,9 & 15,2 & 11,3 & 6,6 & 6,6 & 9,9 & 8,6 & 15,2 & 3,3 & & \\
\hline
\end{tabular}

Dargestellt sind die Häufigkeiten von Ratingmeldungen, differenziert nach Jahren und Ratingkategorien. Die Meldungen enthalten Herauf- respektive Herabstufungen sowie angekündigte Ratingüberprüfungen. Die Kategorien A, Baa und Ba enthalten jeweils die drei Stufen dieser Kategorie, auch die mit 1, 2 und 3 ergänzten. Die Kategorien AAA - Aa und B - C enthalten alle Ratingstufen dieser Bereiche. Das Symbol $\%$ bezeichnet den prozentualen Anteil an der Gesamtanzahl der Ratingmeldungen.

Tabelle 3: Verteilung der Ausgangsratings der Ratingmeldungen über den Beobachtungszeitraum

Tabelle 4 zeigt die Ratingmeldungen differenziert nach den Begründungskategorien. Der Anteil der Meldungen, die auf Kapitalstrukturänderungen zurückzuführen sind, beträgt im Durchschnitt 28,5\%. Der Anteil performancebedingter Meldungen beläuft sich hingegen auf $71,5 \%$. Dabei zielen 62,2\% der Meldungen auf Ratingänderungen ab, während $37,2 \%$ durch Ratingüberprüfungen begründet werden. 


\begin{tabular}{l|ccccccccccc|c|}
\hline & \multicolumn{1}{c}{ Ratingänderung } & \multicolumn{1}{c}{ Ratingüberprüfung } \\
\cline { 2 - 13 } & Down & Up & Summe & $\%$ & Down & Up & Summe & $\%$ & Summe & $\%$ \\
\hline Performance & 49 & 17 & 66 & 70,2 & 37 & 5 & 42 & 73,7 & 108 & 71,5 \\
Kapitalstruktur & 19 & 9 & 28 & 29,8 & 9 & 6 & 15 & 26,3 & 43 & 28,5 \\
\hline Summe & 68 & 26 & 94 & & 46 & 11 & 57 & & 151 & \\
\hline$\%$ & 45,0 & 17,2 & & 62,2 & 30,5 & 7,3 & & 37,8 & & 100 \\
\hline
\end{tabular}

Die Tabelle stellt die Anzahl der Ratingmeldungen der Ratingarten differenziert nach der zugrundeliegenden Begründungskategorie dar.

Tabelle 4: Anzahl der Ratingänderungen und Ratingüberprüfung nach Ratingbegründung

\section{Empirische Ergebnisse}

\subsection{Ergebnisse der univariaten Analyse}

Tabelle 5 zeigt die Analyseergebnisse zu Hypothese 1 für die Ereignisfenster [0, +1], [-1, +1] sowie $[-2,+2]$ differenziert nach Art der Meldung (Ratingänderung versus Ratingüberprüfung). Dabei zeigt sich, dass bei einer pauschalen Beurteilung von Ratingmeldungen also ohne Berücksichtigung des Grundes - keine signifikanten Einflüsse auf die abnormale Rendite festgestellt werden können. So können bei Heraufstufungen weitestgehend keine signifikanten Einflüsse auf die abnormale Rendite festgestellt werden. Allein im Ereignisfenster $[-2,+2]$ kann ein solcher Einfluss auf dem 10\%-Niveau bei Ratingänderungen ausgemacht werden. Der geringe Signifikanzgrad sowie die Tatsache, dass diese Beobachtung in das grösste Zeitfenster fällt, könnten indes alternativ auf andere Effekte zurückzuführen sein. Auch bei herabstufenden Ratingänderungen kann kein signifikanter Einfluss auf die abnormale Rendite ausgemacht werden. Lediglich bei herabstufenden Ratingüberprüfungen können negative Einflüsse festgestellt werden, die bis auf das $1 \%$-Niveau signifikant sind. Konkret können signifikant negative Einflüsse von $-1,27 \%$ bis $-2,64 \%$ nachgewiesen werden.

\begin{tabular}{|c|c|c|c|c|c|c|c|}
\hline & & \multicolumn{2}{|l|}{$[0,+1]$} & \multicolumn{2}{|l|}{$[-1,+1]$} & \multicolumn{2}{|l|}{$[-2,+2]$} \\
\hline \multicolumn{8}{|c|}{ Panel A: Durchschnittliche abnormale Aktienkursreaktionen bei Herabstufungen } \\
\hline \multirow[t]{6}{*}{ Ratingänderung } & $\operatorname{KAR}[\%]$ & $-0,63$ & & $-0,46$ & & 0,15 & \\
\hline & Anteil KAR $<0[\%]$ & 57,35 & & 55.88 & & 51,47 & \\
\hline & $\mathrm{t}$-Test & $-1,4189$ & & $-0,8321$ & & 0,2140 & \\
\hline & Vorzeichentest & 1,0914 & & 0,8489 & & 0,1213 & \\
\hline & Corrado & 0,9535 & & 0,2963 & & $-0,4323$ & \\
\hline & $n$ & 68 & & 68 & & 68 & \\
\hline \multirow[t]{6}{*}{ Ratingüberprüfung } & $\operatorname{KAR}[\%]$ & $-1,27$ & & $-1,84$ & & $-2,64$ & \\
\hline & Anteil KAR < $0[\%]$ & 60,87 & & 60,87 & & 67,39 & \\
\hline & $\mathrm{t}$-Test & $-2,1372$ & $* *$ & $-2,5283$ & $* *$ & $-2,8056$ & $* * *$ \\
\hline & Vorzeichentest & 1,3270 & $*$ & 1,3270 & $*$ & 2,2116 & $* *$ \\
\hline & Corrado & 1,8132 & $*$ & 2,2009 & $* *$ & 2,7269 & $* * *$ \\
\hline & $n$ & 46 & & 46 & & 46 & \\
\hline
\end{tabular}




\begin{tabular}{|c|c|c|c|c|}
\hline & & {$[0,+1]$} & {$[-1,+1]$} & {$[-2,+2]$} \\
\hline \multicolumn{5}{|c|}{ Panel B: Durchschnittliche abnormale Aktienkursreaktionen bei Heraufstufungen } \\
\hline \multirow[t]{6}{*}{ Ratingänderung } & KAR $[\%]$ & 0,38 & 0,47 & 1,05 \\
\hline & Anteil KAR < $0[\%]$ & 53,85 & 46,15 & 50,00 \\
\hline & $\mathrm{t}$-Test & 0,8235 & 0,8299 & 1,4333 \\
\hline & Vorzeichentest & 0,1961 & 0,1961 & $-0,1961$ \\
\hline & Corrado & $-0,9904$ & $-1,1624$ & $-1,8129$ \\
\hline & $n$ & 26 & 26 & 26 \\
\hline \multirow[t]{6}{*}{ Ratingüberprüfung } & $\operatorname{KAR}[\%]$ & $-0,14$ & 0,61 & 0,56 \\
\hline & Anteil KAR < $0[\%]$ & 54,55 & 45,45 & 36,36 \\
\hline & t-Test & $-0,2143$ & 0,7660 & 0,5407 \\
\hline & Vorzeichentest & 0,0000 & 0,0000 & 0,6030 \\
\hline & Corrado & 0,3716 & $-0,2714$ & $-0,3509$ \\
\hline & $n$ & 11 & 11 & 11 \\
\hline \multicolumn{5}{|c|}{$\begin{array}{l}{[0,+1],[-1,+1] \text { und }[-2,+2] \text { bezeichnen die Ereignisfenster, wobei } 0 \text { für den Ereignistag steht. KAR be- }} \\
\text { zeichnet die kumulierte durchschnittliche abnormale Rendite im Ereignisfenster. Anteil KAR < } 0 \text { gibt den } \\
\text { Anteil der negativen kumulierten durchschnittlichen abnormalen Renditen in der jeweiligen Stichprobe an. } \\
\text { Die arithmetischen Mittelwerte (Mediane) werden mithilfe des t-Tests (Vorzeichentest sowie Corrado- } \\
\text { Rangplatzverfahren) auf eine signifikante Abweichung von Null getestet. Die Symbole } * / * * * * * * \text { zeigen die } \\
\text { Signifikanz mit einer Irrtumswahrscheinlichkeit von } 10 \% / 5 \% / 1 \% \text { (zweiseitiger Test) an. }\end{array}$} \\
\hline
\end{tabular}

Tabelle 5: Aktienkursreaktionen auf Ratingänderungen und -überprüfungen

In einem nächsten Schritt ist zu analysieren, ob bei einer Berücksichtigung des Ratinggrundes und damit hinsichtlich der formulierten Hypothesen $1 a$ und $1 b$ signifikante Wirkungszusammenhänge festgestellt werden können. Die Ergebnisse können Tabelle 6 entnommen werden. Konkret werden dort die Einflüsse auf die abnormale Rendite differenziert nach dem Grund für die Ratingänderung (Performance versus Kapitalstruktur) sowie nach der Art der Ratingmeldung (Ratingänderung versus Ratingüberprüfung) dargestellt.

Betrachtet man Herabstufungen differenziert nach Performance und Kapitalstruktur, so sind die prognostizierten, gegenläufigen Effekte festzustellen. Konkret sind bei performancebedingten Herabstufungen signifikant-negative Kursreaktionen festzustellen, während bei kapitalstrukturbedingten Herabstufungen positive Effekte auf die abnormale Rendite wirken. Mit Blick auf die Performance zeigt sich insbesondere bei angekündigten Ratingüberprüfungen ein sehr deutlicher negativer Einfluss von $-1,85 \%$ bis $-3,77 \%$, der fast durchgängig auf einem Signifikanzniveau von $1 \%$ bestätigt werden kann. Wird eine tatsächliche Herabstufung zu einem späteren Zeitpunkt vorgenommen, ist der Einfluss auf die abnormalen Renditen hingegen deutlich geringer. So liegt dieser Effekt lediglich zwischen $-0,48 \%$ und $-1,28 \%$. Zudem kann dieser nur für die beiden kürzeren Ereignisfenster auf einem 5\%-respektive 10\%-Niveau als signifikant nachgewiesen werden. Eine Überprüfung des bestehenden Ratings aufgrund eines zunehmenden Verschuldungsgrades hat einen positiven Einfluss auf die Höhe der abnormalen Rendite von 1,1\% bis 2,01\%. Dieser kann zudem bis auf das 5\%-Niveau als signifikant nachgewiesen werden. Wird das 
Rating zu einem späteren Zeitpunkt tatsächlich geändert, resultieren daraus ebenfalls signifikant-positive Einflüsse auf die abnormale Rendite, die mit 1,03\% bis 1,77\% leicht geringer ausfallen.

Bei Heraufstufungen lassen sich reziproke Ergebnisse feststellen. So wirken performancebedingte Heraufstufungen positiv auf die abnormale Rendite, während kapitalstrukturbedingte Heraufstufungen einen negativen Einfluss haben. Auffällig ist jedoch, dass für beide Fallgruppen keine signifikanten Effekte bei angekündigten Überprüfungen gefunden werden können. Erst bei tatsächlichen Ratingänderungen ist eine solche Signifikanz nachweisbar. So kann bei Performanceverbesserungen ein positiver Effekt von 1,21\% bis zu $2,09 \%$ auf einem Signifikanzniveau von bis zu 1\% festgestellt werden. Bei Änderungen aufgrund der Kapitalstruktur sind Einflüsse zwischen -0,9\% und -1,19\% auf einem Signifikanzniveau von bis zu 5\% auszumachen. Die Insignifikanz bei angekündigten Heraufstufungen könnte mit der geringen Anzahl an Beobachtungen zusammenhängen, da lediglich 5 bzw. 6 Meldungen je Begründungskategorie vorliegen.

\begin{tabular}{|c|c|c|c|c|c|c|c|}
\hline & & \multicolumn{2}{|l|}{$[0,+1]$} & \multicolumn{2}{|l|}{$[-1,+1]$} & \multicolumn{2}{|l|}{$[-2,+2]$} \\
\hline \multicolumn{8}{|c|}{ Panel A: Durchschnittliche abnormale Aktienkursreaktionen bei Herabstufungen } \\
\hline \multirow{6}{*}{$\begin{array}{l}\text { Ratingänderung } \\
\text { Grund: Performance }\end{array}$} & $\operatorname{KAR}[\%]$ & $-1,28$ & & $-1,14$ & & $-0,48$ & \\
\hline & Anteil KAR < 0 [\%] & 67,35 & & 67,35 & & 57,14 & \\
\hline & t-Test & $-2,3668$ & $* *$ & $-1,7232$ & $*$ & $-0,5569$ & \\
\hline & Vorzeichentest & 2,2857 & $* *$ & 2,2857 & $* *$ & 0,8571 & \\
\hline & Corrado & 2,3231 & $* *$ & 1,6272 & & 0,8419 & \\
\hline & $n$ & 49 & & 49 & & 49 & \\
\hline \multirow{6}{*}{$\begin{array}{l}\text { Ratingänderung } \\
\text { Grund: Kapitalstruktur }\end{array}$} & $\operatorname{KAR}[\%]$ & 1,03 & & 1,31 & & 1,77 & \\
\hline & Anteil KAR < $0[\%]$ & 31,58 & & 26,32 & & 36,84 & \\
\hline & $\mathrm{t}$-Test & 1,4953 & & 1,5552 & & 1,6236 & \\
\hline & Vorzeichentest & 1,3765 & $*$ & 1,8353 & $* *$ & 0,9177 & \\
\hline & Corrado & $-1,7415$ & $*$ & $-1,8761$ & $*$ & $-2,0069$ & $* *$ \\
\hline & $n$ & 19 & & 19 & & 19 & \\
\hline \multirow{6}{*}{$\begin{array}{l}\text { Ratingüberprüfung } \\
\text { Grund: Performance }\end{array}$} & KAR [\%] & $-1,85$ & & $-2,58$ & & $-3,77$ & \\
\hline & Anteil KAR < $0[\%]$ & 72,97 & & 70,27 & & 81,08 & \\
\hline & $\mathrm{t}$-Test & $-2,6435$ & $* * *$ & $-3,0206$ & $* * *$ & $-3,4109$ & $* * *$ \\
\hline & Vorzeichentest & 2,6304 & $* * *$ & 2,3016 & $* *$ & 3,6168 & $* * *$ \\
\hline & Corrado & 3,0499 & $* * *$ & 3,2648 & $* *$ & 3,8229 & $* * *$ \\
\hline & $n$ & 37 & & 37 & & 37 & \\
\hline \multirow{6}{*}{$\begin{array}{l}\text { Ratingüberprüfung } \\
\text { Grund: Kapitalstruktur }\end{array}$} & KAR [\%] & 1,10 & & 1,21 & & 2,01 & \\
\hline & Anteil KAR < $0[\%]$ & 11,11 & & 22,22 & & 11,11 & \\
\hline & $\mathrm{t}$-Test & 1,2008 & & 1,0855 & & 1,3898 & \\
\hline & Vorzeichentest & 2,0000 & $* *$ & 1,3333 & $*$ & 2,0000 & $* *$ \\
\hline & Corrado & $-2,0843$ & $* *$ & $-1,6121$ & & $-1,5555$ & \\
\hline & $n$ & 9 & & 9 & & 9 & \\
\hline
\end{tabular}




\section{$[0,+1] \quad[-1,+1] \quad[-2,+2]$}

Panel B: Durchschnittliche abnormale Aktienkursreaktionen bei Heraufstufungen

\begin{tabular}{|c|c|c|c|c|c|c|c|}
\hline \multirow{6}{*}{$\begin{array}{l}\text { Ratingänderung } \\
\text { Grund: Performance }\end{array}$} & KAR [\%] & \multicolumn{2}{|l|}{1,21} & \multicolumn{2}{|l|}{1,68} & \multicolumn{2}{|l|}{2,09} \\
\hline & Anteil KAR < 0 [\%] & 41,18 & & 29,41 & & 35,29 & \\
\hline & $\mathrm{t}$-Test & 1,9121 & $*$ & 2,1574 & $* *$ & 2,0850 & $* *$ \\
\hline & Vorzeichentest & 0,4851 & & 1,4552 & $*$ & 0,9701 & \\
\hline & Corrado & $-2,1177$ & $* *$ & $-2,4272$ & $* *$ & $-2,6912$ & $* * *$ \\
\hline & $n$ & 17 & & 17 & & 17 & \\
\hline \multirow{6}{*}{$\begin{array}{l}\text { Ratingänderung } \\
\text { Grund: Kapitalstruktur }\end{array}$} & KAR [\%] & $-1,18$ & & $-1,80$ & & $-0,90$ & \\
\hline & Anteil KAR < $0[\%]$ & 77,78 & & 77,78 & & 77,78 & \\
\hline & $\mathrm{t}$-Test & $-1,7909$ & $*$ & $-2,2222$ & $* *$ & $-0,8649$ & \\
\hline & Vorzeichentest & 1,3333 & $*$ & 1,3333 & $*$ & 1,3333 & $*$ \\
\hline & Corrado & 1,2853 & & 1,4364 & & 0,6875 & \\
\hline & $n$ & 9 & & 9 & & 9 & \\
\hline \multirow{6}{*}{$\begin{array}{l}\text { Ratingüberprüfung } \\
\text { Grund: Performance }\end{array}$} & KAR [\%] & 0,36 & & 1,83 & & 1,49 & \\
\hline & Anteil KAR < 0 [\%] & 20,00 & & 20,00 & & 20,00 & \\
\hline & $\mathrm{t}$-Test & 0,3105 & & 1,3012 & & 0,8238 & \\
\hline & Vorzeichentest & 0,8944 & & 0,8944 & & 0,8944 & \\
\hline & Corrado & $-0,7881$ & & $-1,1835$ & & $-0,7675$ & \\
\hline & $n$ & 5 & & 5 & & 5 & \\
\hline \multirow{6}{*}{$\begin{array}{l}\text { Ratingüberprüfung } \\
\text { Grund: Kapitalstruktur }\end{array}$} & KAR [\%] & $-0,55$ & & $-0,40$ & & $-0,22$ & \\
\hline & Anteil KAR < $0[\%]$ & 83,33 & & 66,67 & & 50,00 & \\
\hline & $\mathrm{t}$-Test & $-0,7846$ & & $-0,4668$ & & $-0,2010$ & \\
\hline & Vorzeichentest & 1,2247 & & 0,4082 & & $-0,4082$ & \\
\hline & Corrado & 1,1635 & & 0,6894 & & 0,2249 & \\
\hline & $n$ & 6 & & 6 & & 6 & \\
\hline
\end{tabular}

$[0,+1],[-1,+1]$ und $[-2,+2]$ bezeichnen die Ereignisfenster, wobei 0 für den Ereignistag steht. KAR bezeichnet die kumulierte durchschnittliche abnormale Rendite im Ereignisfenster. Anteil KAR $<0$ gibt den Anteil der negativen kumulierten durchschnittlichen abnormalen Renditen in der jeweiligen Stichprobe an. Die arithmetischen Mittelwerte (Mediane) werden mithilfe des t-Tests (Vorzeichentest sowie CorradoRangplatzverfahren) auf eine signifikante Abweichung von Null getestet. Die Symbole */**/*** zeigen die Signifikanz mit einer Irrtumswahrscheinlichkeit von 10\%/5\%/1\% (zweiseitiger Test) an.

Tabelle 6: Aktienkursreaktionen auf Ratingänderungen und -überprüfungen differenziert nach Begründung der Ratinganpassung

Zusammenfassend können die theoretisch abgeleiteten Wirkungsweisen der Optionspreistheorie nach Merton sowohl mit Blick auf Veränderungen der Kapitalstruktur als auch 
hinsichtlich der Performance bestätigt werden. Die erforderliche Signifikanz bei angekündigten Überprüfungen kann jedoch nur bei Herabstufungen nachgewiesen werden. Bis auf diese Ausnahme werden die formulierten Hypothesen $2 a$ und $2 b$ daher vollständig gestützt.

\subsection{Multivariate Analyse}

\subsubsection{Operationalisierung der Hypothesen}

Neben den formulierten Hypothesen $1 a$ und $1 b$ sollen weitere Einflüsse auf die abnormale Rendite auf Basis einer multiplen linearen Regression analysiert werden. Folglich sind in einem ersten Schritt die formulierten Hypothesen durch unabhängige Variablen zu operationalisieren, bevor in einem zweiten Schritt Kontrollvariablen hinzugefügt werden.

Auch im multivariaten Modell sollen die Hypothesen $1 a$ und $1 b$ dahingehend überprüft werden, ob Änderungen der Kapitalstruktur gegenläufig im Vergleich zu Änderungen aufgrund der Unternehmensperformance auf die abnormale Rendite wirken. Dafür wird eine kategoriale Variable „KAPITALSTR“ in die Regressionsgleichung eingefügt, die den Wert 1 zugewiesen bekommt, wenn die Ratingänderung durch die Kapitalstruktur bedingt wird bzw. ansonsten den Wert 0 anzeigt.

Nach Hypothese 2 soll die Höhe der abnormalen Rendite u. a. davon abhängen, ob der Ratingänderung eine Ankündigung zur Überprüfung vorausgegangen ist, so dass der Kapitalmarkt mögliche Effekte eventuell bereits im Vorfeld antizipiert hat. Dies wird mit der kategorialen Variablen „ÜBERPRÜF“ in der Regressionsgleichung umgesetzt, die den Wert 1 annimmt, wenn einer Ratingänderung eine solche Ankündigung vorausgegangen ist bzw. ansonsten den Wert 0 aufweist. Bei der hier betrachteten Stichprobe fand in 63,2\% der Fälle eine solche Ratingüberprüfung statt, während folglich 36,8\% aller Ratingänderungen unangekündigt waren.

Mit der unabhängigen Variablen „RATING_Alt“ wird das Rating berücksichtigt, welches vor der Ratingmeldung bestanden hat. So wird in Hypothese 3 vermutet, dass der Kapitalmarkt auf Ratingänderungen sensibler reagiert, wenn das Unternehmen bereits vorher ein verhältnismässig schlechtes Rating aufgewiesen hat. Um das Rating in der Berechnung zu verwenden, muss die Ratingskala Aaa-C in numerische Ratingbeurteilungen transformiert werden. Dazu wird einem Aaa-Rating der Wert 1 zugewiesen, Aa1 eine 2 während ein C-Rating schliesslich den Wert 21 erhält. In der untersuchten Stichprobe beträgt das nummerische Rating im Mittel 8,185, was annähernd einem Baa1-Rating entspricht. Konkret müsste nach der Hypothese gelten, dass je höher die zugewiesene Nummer ist, desto höher müsste der Effekt auf die abnormale Rendite sein. Es dürfte jedoch fraglich sein, ob die zusätzliche Bedeutung linear mit den Ratingnummern ansteigt. Folglich wird hier neben einem linear ansteigenden Verlauf auch eine abnehmende respektive ansteigende zusätzliche Bedeutung der Ratingnummer geprüft, indem für einen degressiven Zusammenhang alternativ die Wurzel sowie der Logarithmus Naturalis der Ratingnummer verwendet wird, bzw. für eine zunehmende Grenzbedeutung die Ratingnummer quadriert bzw. exponiert wird. Im Ergebnistableau wird jene Variante ausgewiesen, die dem Modell den höchsten zusätzlichen Erklärungsgehalt liefert.

Darüber hinaus soll für verschiedene weitere Einflussfaktoren kontrolliert werden. So könnte vermutet werden, dass der Kapitalmarkt umso stärker reagiert, je mehr das ursprüngliche Rating geändert wurde. Wird bspw. ein Rating lediglich um eine Ratingklasse 
verschlechtert, sollte der Einfluss auf die abnormale Rendite geringer ausfallen, als wenn ein Rating um drei Klassen geändert wird. Allgemein dürfte also gelten, dass der Kapitalmarkt bei umfangreicheren Ratingänderungen den Wert des Fremdkapitals deutlicher korrigiert als bei kleineren Anpassungen. Folglich sollte auch der Marktwert des Eigenkapitals entsprechend umfänglicher schwanken, was zu höheren Einflüssen auf die abnormale Rendite bei umfangreichen Ratingänderungen führen dürfte. ${ }^{3}$ Konkret wird eine Kontrollvariable „ $\triangle \mathrm{RATING“} \mathrm{eingefügt,} \mathrm{die} \mathrm{die} \mathrm{Anzahl} \mathrm{der} \mathrm{geänderten} \mathrm{Ratingstufen} \mathrm{angibt.} \mathrm{Dafür}$ wird die Differenz aus jener Ratingnummer gebildet, die dem Unternehmen vor der Ratingänderung zugewiesen werden konnte, und jenem numerischen Wert, den das Unternehmen nach der Ratingänderung aufweist. Im Durchschnitt wurden dabei 1,211 Ratingstufen geändert und maximal umfassen die Änderungen drei Stufen. Veränderungen, die mehr als zwei Stufen umfassen, betreffen ausschliesslich Herabstufungen.

Mit Blick auf eine mögliche Risikoaversion soll zudem kontrolliert werden, ob Anleger der Investment Grade-Grenze eine besondere Bedeutung beimessen. Eigenkapitalgeber könnten beim Unterschreiten der Investment Grade-Grenze - bei Moody's ab Ba1 - relativ stärker dazu neigen, ihr Engagement im betroffenen Unternehmen zu beenden, et vice versa. Es soll kontrolliert werden, ob die abnormale Rendite bei jenen Rating-Änderungen höher ist, die zu einer Über- bzw. Unterschreitung der Investment Grade-Grenze führen (Holthausen/Leftwich 1986, 74-80). Dafür wird eine kategoriale Variable „IG_GRENZE“ eingefügt, die den Wert 1 annimmt, wenn es zu einer solchen Durchbrechung kommt. In allen anderen Fällen wird ihr der Wert 0 zugewiesen. Bei 9,3\% der Ratingänderungen wird diese Grenze durchbrochen.

Auf den internationalen Kapitalmärkten agieren zahlreiche Informationsintermediäre, die zum Abbau von Informationsasymmetrien zwischen Kapitalgebern und Kapitalnehmern beitragen. Ratingagenturen sind nur eine dieser Instanzen. Der Informationsbeitrag, den Ratingmeldungen leisten können, hängt folglich massgeblich vom Informationsniveau der Eigenkapitalgeber ab. Je höher dieser Informationsbeitrag ist, desto höher könnte die abnormale Rendite ausfallen. Verfügen hingegen die Eigenkapitalgeber bereits über entsprechende Informationen, so dürften Ratingmeldungen die abnormale Rendite lediglich begrenzt beeinflussen. Insgesamt könnte also unterstellt werden, dass die Fähigkeit der Agenturen, neue Informationen am Kapitalmarkt zu platzieren, mit zunehmender Anzahl weiterer gut informierter Intermediäre abnimmt. Als Indikator für die Anzahl dieser Intermediäre kann für die Anzahl an Analysten kontrolliert werden, die das entsprechende Unternehmen beobachten (Pongspaibool 1991, 123 f.). Konkret wird dabei jene Anzahl an Analysten verwendet, die den jährlichen Gewinn pro Aktie prognostizieren. Diese Anzahl liegt im Mittel bei 22,364, wobei ein Unternehmen stets mindestens von einem und maximal von 44 Analysten beobachtet wird. Dabei wird ein abnehmender Grenzeinfluss je zusätzlichem Analysten auf die Höhe der abnormalen Rendite vermutet, was über den Logarithmus Naturalis aus der Analystenanzahl umgesetzt wird.

Bei der Diskussion um die Optionspreistheorie in Abschnitt 2.1 wird erörtert, dass neben den Einflussfaktoren Performance und Kapitalstruktur zudem die Volatilität des Unternehmenswertes den Wert des Eigen- bzw. des Fremdkapitals beeinflussen. Während die beiden erstgenannten Einflüsse mit der Hypothese 1a bzw. $1 b$ geprüft werden, soll für die

3 Jorion/Zhang (2007) zeigen sogar in ihrer Studie, dass sich der Marktwert des Eigenkapitals nicht linear je Ratingstufe sondern überproportional je Stufe ändert. 
Volatilität kontrolliert werden. Dafür wird der $\beta$-Faktor aus der Berechnung der abnormalen Rendite als Variable BETA in das Modell integriert.

Aus diesen unabhängigen Variablen nebst Kontrollvariablen lässt sich beispielhaft für das Untersuchungsfenster $[0 ;+1]$ die folgende Regressionsgleichung ableiten:

$$
\begin{aligned}
& \overline{K_{A R}}=\text { Konstante }+y_{1} \text { KAPITALSTR }+y_{2} \ddot{U B E R P R U ̈ F}+y_{3} R A T I N G \_A l t+y_{4} I G-G r e n- \\
& z e+y_{5} \Delta R A T I N G+y_{6} \ln (A N A L Y S T E N)+y_{7} B E T A F A K T O R+\varepsilon_{\mathrm{R}}
\end{aligned}
$$

mit: $\quad \gamma_{i} \quad$ Regressionskoeffizient der Variable i

$\varepsilon_{R} \quad$ Störterm des Regressionsmodells

\subsubsection{Ergebnisse}

Im Folgenden sollen die Einflüsse dieser verschiedenen unabhängigen Variablen und damit die eingangs formulierten Hypothesen überprüft bzw. für die Kontrollvariablen kontrolliert werden. So können die Hypothesen $1 a$ und $1 b$ zum Zusammenhang der Ratingbegründung mit Ratingänderungen auch im multivariaten Kontext bestätigt werden. Es kann bei kapitalstrukturbedingten Herabstufungen ein zwischen 0,022\% und 0,035\% positiver Einfluss auf einem Signifikanzniveau von bis zu 1\% nachgewiesen werden. Bei Heraufstufungen zeigt sich hingegen ein genau reziproker Effekt. So werden Heraufstufungen aufgrund einer erhöhten Eigenkapitalausstattung mit negativen Einflüssen zwischen -0,024\% und $-0,034 \%$ auf die abnormale Rendite belegt, deren Signifikanz ebenfalls bis auf ein Niveau von $1 \%$ festgestellt werden kann. Folglich können die aus dem theoretischen Modell abgeleiteten Zusammenhänge auch im multivariaten Kontext bestätigt werden.

Die Hypothese, dass die Höhe der abnormalen Rendite davon beeinflusst wird, ob der Ratingänderung eine entsprechend angekündigte Überprüfung vorausging, kann partiell bestätigt werden. Ist einer Herabstufung eine entsprechende Ankündigung vorausgegangen, so hat dies einen abschwächenden Einfluss auf die abnormale Rendite. Konkret kann auf einem Signifikanzniveau von bis zu 5\% gezeigt werden, dass in einem solchen Fall die abnormale Rendite zwischen 0,026\% und 0,045\% geringer ist. Bei Heraufstufungen kann hingegen weder eine eindeutige noch eine signifikante Wirkungsrichtung festgestellt werden. Entsprechend antizipiert der Kapitalmarkt angekündigte Überprüfungen bei Herabstufungen stärker. Ankündigungen im Kontext von Heraufstufungen sind für Anteilseigner dagegen weniger bedeutend. Dies stimmt mit dem univariaten Ergebnis überein, wonach angekündigten Überprüfungen hinsichtlich einer möglichen Heraufstufungen ebenfalls keinen signifikanten Erklärungsbeitrag zur abnormalen Rendite liefern können. Hypothese 2 kann folglich nur bei Herabstufungen gestützt werden.

Mit Blick auf die Bedeutung des Ausgangsratings wurde Hypothese 3 aufgestellt, dass je schlechter das Ausgangsrating des Unternehmens ist, umso eher veräussern Eigenkapitalgeber ihre Anteile, was negativ auf die abnormale Rendite wirkt. Die Ergebnisse weisen diesen vermuteten Effekt in Höhe von $-0,001 \%$ bis $-0,003 \%$ auf einem Signifikanzniveau von bis zu 5\% nach. Eine reziproke Wirkung bei Heraufstufungen kann hingegen nicht festgestellt werden. Hier zeigen die Berechnungen weder eindeutige noch signifikante Wirkungszusammenhänge. Folglich kann auch Hypothese 3 nur für Herabstufungen gestützt werden. 


\begin{tabular}{|c|c|c|c|c|c|c|c|c|c|c|}
\hline \multirow{3}{*}{$\overline{\text { ÜBERPRÜF }}$} & \multicolumn{6}{|c|}{ Herabstufungen } & \multicolumn{4}{|c|}{ Heraufstufungen } \\
\hline & \multicolumn{2}{|c|}{$[0,+1]$} & \multicolumn{2}{|c|}{$[-1,+1]$} & \multicolumn{2}{|c|}{$[-2,+2]$} & {$[0,+1]$} & {$[-1,+1]$} & \multicolumn{2}{|l|}{$[-2,+2]$} \\
\hline & $-0,026$ & $*$ & $-0,026$ & $*$ & $-0,045$ & $* *$ & 0,004 & 0,018 & $-0,017$ & \\
\hline & $(-1,80)$ & & $(-1,95)$ & & $(-2,40)$ & & $(-0,19)$ & $(0,71)$ & $(-0,50)$ & \\
\hline \multirow[t]{2}{*}{ KAPITALSTR } & 0,022 & $* * *$ & 0,024 & $* *$ & 0,035 & $* * *$ & $-0,024 * * *$ & $-0,034 * *$ & $-0,025 *$ & \\
\hline & $(2,81)$ & & $(2,62)$ & & $(2,81)$ & & $(-3,08)$ & $(-2,60)$ & $(-1,85)$ & \\
\hline \multirow[t]{2}{*}{ RATING_ALT } & $-0,002$ & $* *$ & $-0,003$ & $* *$ & $-0,001$ & $* *$ & $-0,002$ & 0,001 & 0,002 & \\
\hline & $(-2,68)$ & & $(-2,62)$ & & $(-1,17)$ & & $(-1,41)$ & $(0,84)$ & $(0,81)$ & \\
\hline \multirow[t]{2}{*}{ IG_GRENZE } & 0,010 & & 0,003 & & 0,001 & & 0,009 & 0,006 & 0,005 & \\
\hline & $(1,02)$ & & $(0,29)$ & & $(-0,05)$ & & $(1,33)$ & $(0,65)$ & $(0,54)$ & \\
\hline \multirow[t]{2}{*}{$\triangle \mathrm{RATING}$} & 0,016 & & 0,011 & & 0,015 & & 0,007 & 0,011 & $-0,014$ & \\
\hline & $(1,26)$ & & $(1,04)$ & & $(0,97)$ & & $(0,40)$ & $(0,55)$ & $(-0,66)$ & \\
\hline \multirow[t]{2}{*}{$\ln ($ ANALYST) } & 0,008 & $* *$ & 0,008 & & 0,007 & & 0,002 & 0,004 & 0,006 & \\
\hline & $(2,70)$ & & $(1,68)$ & & $(0,91)$ & & $(0,50)$ & $(0,63)$ & $(1,29)$ & \\
\hline \multirow[t]{2}{*}{ Beta } & 0,005 & & 0,002 & & 0,023 & $*$ & 0,015 & 0,007 & 0,000 & \\
\hline & $(1,00)$ & & $(0,23)$ & & $(1,83)$ & & $(0,93)$ & $(0,31)$ & $(0,00)$ & \\
\hline \multirow[t]{2}{*}{ Konstante } & $-0,031$ & $* * *$ & $-0,028$ & $* *$ & $-0,062$ & $* *$ & 0,012 & $-0,001$ & $-0,017$ & \\
\hline & $(-4,1)$ & & $(-2,44)$ & & $(-2,29)$ & & $(0,82)$ & $(-0,03)$ & $(-0,57)$ & \\
\hline $\mathrm{n}$ & 114 & & 114 & & 114 & & 37 & 37 & 37 & \\
\hline Adjustiertes $\mathrm{R}^{2}$ & 0,1791 & & 0,1519 & & 0,1528 & & 0,1254 & 0,1039 & $-0,0438$ & \\
\hline F-Statistik & 4,52 & $* * *$ & 4,33 & $* *$ & 4,07 & $* * *$ & 1,78 & 2,17 & 3,11 & $* *$ \\
\hline
\end{tabular}

Die Tabelle zeigt die Koeffizienten der OLS-Regression mit der abhängigen Variablen der abnormalen Rendite für die Ereignisfenster $[0,+1],[-1,+1]$ und $[-2,+2]$. Die abhängigen Variablen werden jeweils mittels des Marktmodells (MM) bestimmt. Die t-Statistik wird in Klammern berichtet. Da die 114 bzw. 37 Ankündigungen nur auf 38 Unternehmen basieren, beruht die t-Statistik auf cluster-robusten Standardfehlern, welche korrelierte Fehlerterme innerhalb der Unternehmen berücksichtigen.

Der Dummy-Variablen ÜBERPR ÜF wird der Wert 1 zugewiesen, wenn eine Ratingüberprüfung angekündigt wird (Ratingänderung $=0$ ). Die Variable RATING_ALT gibt die Ratingnummer vor der Ratingmeldung an. KAPITALSTR bezeichnet eine Dummy-Variable, die berücksichtigt, ob als Begründung für eine Ratingmeldung die Kapitalstruktur genannt wird $(1=\mathrm{ja}, 0=$ nein). IG_GRENZE ist die Bezeichnung der Dummy-Variable für Ratingänderungen, die die Investment Grade-Grenze durchbrechen, bzw. bei Reviews, wenn die Überschreitung dieser Grenze droht. $\triangle$ RATING ist die Variable für die Anzahl der veränderten Ratingstufen. Sie berechnet sich aus dem Rating vor der Meldung minus dem Rating nach der Meldung. Bei Ratingüberprüfungen wird die Mindestanzahl verwendet. $\ln (A N A L S T)$ steht für den Logarithmus der Anzahl der Analysten, welche das jeweilige Unternehmen beurteilen.

Die Symbole $* / * * * * *$ zeigen die Signifikanz mit einer Irrtumswahrscheinlichkeit von $10 \% / 5 \% / 1 \%$ (zweiseitiger Test) an.

Alle VIFs $<1,5$.

Tabelle 7: Regressionsanalyse von Einflussfaktoren der abnormalen Rendite

Ferner wurde für verschiedene weitere Variablen kontrolliert, die jedoch nur partiell einen signifikanten Einfluss auf die abnormale Rendite bei wenigen Ereignisfenstern haben. Mit Blick auf die Bedeutung eines Über- bzw. Unterschreitens einer Investment Grade-Grenze 
zeigt sich, dass weder eindeutige noch signifikante Wirkungszusammenhänge gefunden werden können. Folglich scheinen die für Fremdkapitalgeber formulierten Investitionsempfehlungen nicht unmittelbar auf das Anlageverhalten der Eigenkapitalgeber auszustrahlen. Hinsichtlich der Anzahl der Stufen, die durch die Ratingmeldung geändert wurden, lässt sich grundsätzlich sowohl für Herauf- als auch für Herabstufungen der angenommene positive Zusammenhang zeigen. So gilt, dass je mehr Stufen geändert werden, desto stärker ist der Einfluss auf die abnormale Rendite. Dieser Einfluss kann jedoch in keinem Ereignisfenster als signifikant nachgewiesen werden. Die Anzahl der beobachtenden Analysten hat bei Herabstufungen einen positiven Einfluss von 0,007\% bis 0,008\% auf die Höhe der abnormalen Rendite, der jedoch nur in einem Ereignisfenster signifikant auf dem 5\%-Niveau ist. Bei Heraufstufungen lässt sich ebenfalls ein positiver, wenngleich marginaler und nicht signifikanter, Einfluss feststellen. Der Betafaktor hat sowohl bei Herauf- als auch bei Herabstufungen einen positiven Einfluss auf die abnormale Rendite, wenngleich dieser Zusammenhang nur im Ereignisfenster [-2, +2] als signifikant auf dem $10 \%$-Niveau nachgewiesen werden kann.

\section{Diskussion und Zusammenfassung der Befunde}

Bei der zusammengefassten Analyse von Ratingmeldungen kann gezeigt werden, dass bei Heraufstufungen weder bei Ratingänderungen noch bei Ratingüberprüfungen signifikante Einflüsse auf die Höhe der abnormalen Rendite festzustellen sind. Bei Herabstufungen kann ein signifikanter Einfluss nur bei Ratingüberprüfungen abgeleitet werden. Aus diesen Ergebnissen kann jedoch nicht geschlussfolgert werden, dass Informationen, die für Fremdkapitalgeber am Kapitalmarkt platziert werden, für Eigenkapitalgeber nicht wertrelevant seien. Vielmehr zeigt sich, dass abhängig vom Grund neutralisierende Einflüsse auf die abnormale Rendite wirken. So können zwei Sachverhalte festgestellt werden, die die abnormale Rendite reziprok beeinflussen. Konkret wird ein steigender Verschuldungsgrad ceteris paribus von Anteilseignern positiv gewertet, während performancebedingte Herabstufungen auch von Eigenkapitalgebern mit Kursabschlägen bestraft werden, et vice versa. Dies kann in der vorliegenden Studie sowohl im univariaten als auch im multivariaten Kontext mit Blick auf die Wirkungsrichtung voll und hinsichtlich der Signifikanz grösstenteils bestätigt werden. Damit ergänzen die hier ermittelten Ergebnisse die bestehenden Forschungsergebnisse. So können bspw. Gob/Ederington (1993) keine signifikant positiven Reaktionen für Herabstufungen der Kategorie Kapitalstruktur und Heraufstufungen der Kategorie Performance bestätigen. Imbierowicz/Wahrenburg (2009) können signifikant positive Reaktionen für Überprüfungen auf Herabstufungen der Kategorie Kapitalstruktur nach einem nicht-parametrischen und einem parametrischen Test bestätigen. Gropp und Richards (2001) kommen bei Upgrades zu insignifikanten positiven Reaktionen für beide Begründungen.

Bei einer differenzierten Betrachtung von Herauf- respektive Herabstufungen zeigen die Untersuchungsergebnisse zudem, dass angekündigte Ratingüberprüfungen nur bei Herabstufungen zu abnormalen Renditen führen. Solche Ankündigungen hinsichtlich einer möglichen Heraufstufung haben hingegen, bei kleiner Fallzahl, weder bei der univariaten Analyse noch im multivariaten Kontext einen signifikanten Einfluss auf die Höhe der abnormalen Rendite ergeben. Dies steht im Einklang mit den Ergebnissen der Studie von Holthausen/Leftwich (1986) sowie Schweitzer/Szewczyk/Varma (1992), wenngleich letztgenannte keine Signifikanzen nachweisen können (Holthausen/Leftwich 1986, 74-80; Cor- 
nell/Landsman/Shapiro 1989, 468-470). Diese Zurückhaltung bei Heraufstufungen könnte auf zwei unterschiedliche Sachverhalte zurückzuführen sein. Zum einen könnte intuitiv argumentiert werden, dass Kapitalmarktteilnehmer aufgrund einer gewissen Risikoaversion dazu neigen, positive Entwicklungen erst dann in den Unternehmenswert einzupreisen, wenn diese durch eine tatsächliche Ratingänderung verifiziert werden. Diese mögliche Risikoaversion könnte zudem durch das Verhalten bei Ratingüberprüfungen im Kontext der Herabstufung untermauert werden. So haben bereits angekündigte Ratingüberprüfungen bei Herabstufungen einen signifikanten Einfluss auf die abnormale Rendite. Dieser auf den ersten Blick plausiblen Argumentation ist jedoch entgegenzustellen, dass angekündigte Herabstufungen aufgrund eines steigenden Verschuldungsgrades einen positiven Einfluss auf die abnormale Rendite haben. Folglich honorieren Eigenkapitalgeber bereits die Vermutung, dass die Volatilität des Eigenkapitalmarktwertes aufgrund einer Erhöhung des Verschuldungsgrades gestiegen ist mit einem steigenden Börsenkurs, was der formulierten These zur Risikoaversion entgegenstehen dürfte. Folglich scheint über die Risikoaversion nur schwerlich eine stichhaltige Erklärung der Wirkungszusammenhänge möglich. Zum anderen könnte gemutmasst werden, dass der Informationswert von Ratingmeldungen zu Heraufstufungen für den Kapitalmarkt gering sind, da Unternehmen bestrebt sein dürften, positive Entwicklungen unmittelbar zu kommunizieren (Holthausen/Leftwich 1986, 71). Mit Blick auf die reziproke Wirkung von Kapitalstrukturbedingten Ratingänderungen sollten Unternehmen jedoch kritisch analysieren, ob sie diese Kommunikationsstrategie bei Verringerung des Verschuldungsgrades künftig beibehalten möchten.

Die analytische Betrachtung der Ergebnisse zeigt, dass Ratingagenturen zwar einerseits eine exponierte Stellung und in der Öffentlichkeit eine gewichtige Stimme bei der Kommunikation von für den Unternehmenswert relevanten Ereignissen haben. Jedoch nehmen Investoren die kommunizierten Inhalte sehr differenziert auf. Die Ergebnisse der vorliegenden Studie können daher zwar eine nicht marktgerechte Manipulation durch die Stellung von Ratingagenturen mitnichten ausschliessen, relativieren jedoch den ursächlichen Einfluss von Ratingagenturen. Die differenzierte Aufnahme der Informationen deutet vielmehr darauf hin, dass wertbegründende Ereignisse selbst Anlass für Unternehmenswertänderungen sind - Informationen von Ratingagenturen reflektieren diese naturgemäss.

Hinsichtlich der Limitationen der Studie ist zunächst anzuführen, dass die Einteilung von Ratingmeldungen in die Kategorie Performance bzw. Kapitalstruktur mit subjektiven Ermessensspielräumen behaftet sind. Ferner ist die Stichprobe stellenweise relativ klein, wenngleich der Corrado-Test zur Überprüfung signifikanter Zusammenhänge auch für entsprechend geringe Untersuchungsobjekte geeignet ist. Abschliessend ist zu diskutieren, auf welche Sachverhalte die Untersuchungsergebnisse übertragen werden können. So unterliegt die Stichprobe einem selection bias, da mit den Unternehmen des HDAX nur vergleichsweise grosse Unternehmen betrachtet werden. Dabei ist jedoch zu beachten, dass vor allem jene Unternehmen über Ratings verfügen, die Anleihen am Markt emittiert haben. Um einhergehende Emissionskosten zu amortisieren, müssen diese Unternehmen eine gewisse Mindestgrösse überschritten haben. Entsprechend sind die hier untersuchte Forschungsfrage nur für vergleichsweise grosse Unternehmen einschlägig, was eine möglicherweise grössenmässige Verzerrung der Stichprobe und damit eine eingeschränkte Übertragbarkeit auf hier nicht untersuchte Unternehmen relativiert. Schlussendlich sollte eine entsprechende Reaktion von Anteilseignern nur bei solchen Unternehmen möglich sein, die 
fungible Anteile haben, was für kapitalmarktorientierte Unternehmen mit einem Mindestmass an Streubesitz der Fall ist.

Weiterer Forschungsbedarf besteht zum einen mit Blick auf die aktuelle Staatsschuldenkrise. So lässt diese Krise einen starken Einfluss von Länderratings auf den Shareholder Value der Unternehmen des jeweiligen Landes vermuten. Diese Vermutung könnte mit einer international angelegten Ereignisstudie überprüft werden. Zum anderen wurde bislang noch nicht der Einfluss von Ratingmeldungen auf den Wert der Unternehmen derselben Branche untersucht. So könnte insbesondere vermutet werden, dass performancebedingte Ratingmeldungen auch den Shareholder Value von vergleichbaren Unternehmen beeinflussen.

\section{Literaturhinweise}

Backhaus, K./Erichson, B./Plinke, W./Weiber, R. (2011): Multivariate Analysemethoden - Eine anwendungsorientierte Einführung, 13. Aufl., Berlin u.a.

Ball, R./Brown, P. (1969): An Empirical Evaluation of Accounting Income Numbers, in: Journal of Accounting Research, Jg. 6, S. 159-178.

Bannier, C./Hirsch, C. (2010): The economic function of credit rating agencies - What does the watchlist tell us?, in: Journal of Banking and Finance, Jg. 34, S. 3037-3049.

Brown, S./Warner, J. (1980): Measuring Security Price Performance, in: Journal of Financial Economics, Jg. 8, S. 2205-2258.

Brown, S./Warner, J. (1985): Using Daily Stock Returns: The Case of Event Studies, in: Journal of Financial Economics, Jg. 14, S. 3-31.

Campbell, J./Lo, A./MacKinlay, A. (1997): The Econometrics of Financial Markets, 2. pr., with corrections, Princeton.

Cornell, B./Landsman, W./Shapiro, A. (1989): Cross-Sectional Regularities in the Response of Stock Prices to Bond Rating Changes, in: Journal of Accounting, Auditing \& Finance, Jg. 4, S. 460-479.

Corrado, C. (1989): A Nonparametric Test for Abnormal Security-Price Performance in Event Studies, in: Journal of Financial Economics, Jg. 23, S. 385-395.

Cowan, A. (1992): Nonparametric Event Study Tests, in: Review of Quantitative Finance and Accounting, Jg. 2, S. 343-358.

Dichev, I./Piotroski, J. (2001): The Long-Run Stock Returns Following Bond Ratings Changes, in: The Journal of Finance, Jg. 56, S. 173-203.

Entrup, U. (1995): Kapitalmarktreaktionen auf Optionsanleihen, Finanzierungstheoretische Begründung und empirische Analyse der Aktienkursreaktionen, Wiesbaden.

Fama, E./Fisher, L./Jensen, M./Roll, R. (1969): The Adjustment of Stock Prices to New Information, in: International Economic Review, Jg. 10, S. 1-21.

Gerpott, T. (2009): Ereignisstudie, in: Baumgarth, C./Eisend, M./Evanschitzky, H. (Hrsg.): Empirische Mastertechniken, Wiesbaden, S. 203-234.

Goh, J./Ederington, L. (1993): Is a Bond Rating Downgrade Bad News, Good News, or No News for Stockholders?, in: The Journal of Finance, Jg. 48, S. 2001-2008.

Goh, J./Ederington, L. (1999): Cross-Sectional Variation in the Stock Market Reaction to Bond Rating Changes, in: The Quarterly Review of Economics and Finance, Jg. 39, S. 101-112. 
Gropp, R./Richards, A. (2001): Rating Agency Actions and the Pricing of Debt and Equity of European Banks: What Can We Infer About Private Sector Monitoring of Bank Soundness?, European Central Bank Working Paper Series, Working Paper No. 76, August 2001.

Hirshleifer, J. (1970): Investment, Interest and Capital, Englewood Cliffs.

Holthausen, R./Leftwich, R. (1986): The Effect of Bond Rating Changes on Common Stock Prices, in: Journal of Financial Economics, Jg. 17, S. 57-89.

Imbierowicz, B./Wabrenburg, M. (2009): The Impact of Reasons for Credit Rating Announcements in Equity and CDS Markets, Working Paper, Frankfurt (Main).

Jorion, P./Zhang, G. (2007): Information Effects of Bond Rating Changes: The Role of the Rating Prior to the Announcement, in: Journal of Fixed Income, Jg. 16, S. 45-59.

MacKinlay, A. (1997): Event Studies in Economics and Finance, in: Journal of Economic Literature, Jg. 35, S. 13-39.

McWilliams, A./Siegel, D. (1997): Event Studies in Management Research: Theoretical and Empirical Issues, in: Academy of Management Journal, Jg. 40, S. 626-657.

Merton, R. (1974): On the Pricing of Corporate Debt: The Risk Structure of Interest Rates, in: The Journal of Finance, Jg. 42, S. 449-470.

Norden, L./Weber, M. (2004): Informational Efficiency of Credit Default Swap and Stock Markets: The impact of Credit Rating Announcements, in: Journal of Banking and Finance, Jg. 28, S. 2813-2843.

Pongspaibool, N. (1991): An Analysis of the Information Content of Bond Rating Changes: A Case of Differential Information, University of Northan Taxas, Denton.

Robichek, A./Myers, S. (1965): Optimal financing decisions, Englewood Cliffs.

Röder, K. (1999): Kurswirkungen von Meldungen deutscher Aktiengesellschaften, Lohmar Köln.

Schweitzer, R./Szewczyk, S./Varma, R. (1992): Bond Rating Agencies and Their Role in Bank Market Discipline, in: Journal of Financial Services Research, Jg. 6, S. 249-263.

Wappenschmidt, C. (2009): Ratinganalyse durch internationale Ratingagenturen, Frankfurt (Main).

Wieben, H. (2004): Credit Rating und Risikomanagement - Vergleich und Weiterentwicklung der Analysekonzepte, Wiesbaden. 
Steffen Biermann, Dipl.-Kfm., ist wissenschaftlicher Mitarbeiter am Lehrstuhl für ABWL und Controlling an der Universität Duisburg-Essen.

Anschrift: Universität Duisburg-Essen, Lehrstuhl für ABWL und Controlling, D-47057 Duisburg, Tel.: +049 (0)203/379-4491, Fax: +049 (0)203/379-1694, E-Mail: steffen.biermann@uni-due.de

Corinna Ewelt-Knauer, Dr., ist Akademische Rätin a. Zt. am Institut für Rechnungslegung und Wirtschaftsprüfung der Westfälischen Wilhelms-Universität Münster.

Anschrift: Westfälische Wilhelms-Universität Münster, Institut für Rechnungslegung und Wirtschaftsprüfung, D-48143 Münster, Tel.: +049 (0)251/832-8604, Fax: +049 (0)251/832-8601, E-Mail: corinna.ewelt-knauer@wiwi.uni-muenster.de

Andreas Wömpener, Prof. Dr., ist Inhaber des Lehrstuhls für ABWL und Controlling an der Universität Duisburg-Essen.

Anschrift: Universität Duisburg-Essen, Lehrstuhl für ABWL und Controlling, D-47057 Duisburg, Tel.: +049 (0)203/379-4591, Fax: +049 (0)203/379-1694, E-Mail: andreas.woempener@uni-due.de 